\title{
KRAS mutational concordance between primary and metastatic colorectal adenocarcinoma
}

\author{
PANAGIOTIS PALIOGIANNIS ${ }^{1}$, ANTONIO COSSU ${ }^{1}$, FRANCESCO TANDA $^{1}$, \\ GIUSEPPE PALMIERI $^{2}$ and GRAZIA PALOMBA ${ }^{2}$ \\ ${ }^{1}$ Department of Surgical, Microsurgical and Medical Sciences, University of Sassari, Sassari I-07100; \\ ${ }^{2}$ Institute of Biomolecular Chemistry, Cancer Genetics Unit, National Research Council, \\ Sassari I-07040, Italy
}

Received December 19, 2013; Accepted June 15, 2014

DOI: 10.3892/ol.2014.2411

\begin{abstract}
KRAS mutation analysis is commonly performed on tissue samples obtained from primary colorectal cancers (CRCs). The metastatic lesions of CRC are usually considered as qualitatively similar or even identical to the primary tumors. The aim of this study was to evaluate the spectrum and distribution of $K R A S$ mutations in a large collection of CRCs, while also evaluating the concordance of primary and metastatic lesions among available paired specimens from the same patients. A total of 729 patients with histologically confirmed advanced CRC at the University Hospital and Local Health Unit (Sassari, Italy) were included. Clinical and pathological features were obtained from medical records and/or pathology reports. Formalin-fixed, paraffin-embedded tissue samples were used for mutation analysis. Genomic DNA was isolated using a standard protocol; the coding sequence and splice junctions of exons 2 and 3 in the KRAS gene were screened by direct automated sequencing. Overall, 219 (30\%) KRAS mutations were found; $208(30.1 \%)$ were identified in the 690 primary tumors and $11(28.2 \%)$ in the 39 metastatic tissue samples. Among the 31 (4.3\%) patients who had paired samples of primary CRC and synchronous or asynchronous metastases, 28 (90.3\%) showed consistent mutation patterns between the primary tumors and metastatic lesions. In one case, an additive mutation (Q61L) was found in the metastatic tissue, while two other discrepant cases exhibited a different mutation distribution; $\mathrm{Q} 61 \mathrm{H}$ in the primitive lesion and $\mathrm{G} 13 \mathrm{~V}$ in the metastatic lesion in one case, and a mutated primary tumor (Q61L) and wild-type metastasis in another case. The results of this study confirm that a high concordance exists between the results of KRAS mutation analysis performed in primitive and metastatic CRCs; independent subclones may be generated in a limited amount of patients.
\end{abstract}

Correspondence to: Dr Giuseppe Palmieri, Institute of Biomolecular Chemistry, Cancer Genetics Unit, National Research Council, 3 Traversa La Crucca, Sassari I-07040, Italy

E-mail: gpalmieri@yahoo.com

Key words: colorectal cancer, KRAS gene, mutation analysis

\section{Introduction}

Colorectal cancer (CRC) is a neoplastic disease with one of the highest incidence rates worldwide with $>1,200,000$ cases reported in 2008 (1). CRC is the third most common type of neoplastic disease in males and females, following lung and prostate cancer in males, and breast and cervical cancer in females. CRC also presents the fourth most frequent type of cancer-related mortality following lung, stomach and liver cancer $(1,2)$. An evaluation performed by the National Cancer Institute estimated that the total national expenditure for CRC care amounted to 14.1 billion dollars in 2010 in the USA, accounting for $\sim 12 \%$ of the global health care costs in the country; further evidence exists indicating that these costs will continue to grow in the years to come $(3,4)$.

These figures, along with a persistent tendency of incidence rates to increase, depict the relevance of the disease and explain the enormous efforts that have been made in last decades in all fields of preventive, clinical, surgical and molecular science in order to confront the problem. Great responses and results have been obtained in the management of non-invasive and early-stage invasive disease, particularly with the improvements in multidisciplinary endoscopic, surgical, radiotherapeutic and medical oncology protocols. Conversely, progress in the setting of advanced and/or metastatic disease have been less consistent, and the relative survival remains relatively low $(2,5)$. The role of tumor genetics in this setting has been extremely important, particularly in the development of novel therapeutic strategies, based on specific molecular targets.

The most relevant results of 'targeted therapies' have been obtained through the comprehension of the pathophysiological mechanisms of oncogenesis due to genetic modifications involving the epidermal growth factor receptor (EGFR)-RAS cascade. The EGFR is a transmembrane protein for the epidermal growth factor that explicates its functions through the activation of the RAS protein family (HRAS, KRAS and NRAS). Activated RAS proteins promote cell proliferation through several mechanisms, including constitutive stimulation of the mitogen-activated protein kinases (6). EGFR-targeted agents that compete with EGF for binding to the receptor have been employed in clinical practice, in order 
to reduce cell proliferation (7). Oncogenic RAS activation is due to specific mutations in the kinase regions of the genes, producing a constitutive induction of the phosphorylating function of the RAS proteins which, in turn, promote neoplastic proliferation and markedly reduce the effect of EGFR-targeted agents $(7,8)$. Mutation of KRAS has been demonstrated to act as a predictor of the absence of response to EGFR-targeted agents $(9,10)$. These observations led to a requirement for $K R A S$ mutation analysis to be conducted in all patients with $\mathrm{CRC}$, and it is currently performed routinely in clinical practice. However, response rates to EGFR-targeted agents remain low, accounting for $<20 \%$ of cases (11).

Such a low response rate may be explained on the basis of individual biological behavior or genetic background. In this sense, CRC lesions may arise through acquisition of different molecular alterations; activation of oncogenes which differ from those included in $K R A S$-depending pathways, as well as inactivation of specific tumor suppressor genes (such as $A P C$, p16, p53 and DCC) $(12,13)$ or impairment of mismatch repair genes (including $M L H 1, M S H 2$ and, to a lesser extent, PMS2 and $h M S H 6)$ in the subset of CRCs associated with microsatellite instability (14). Recently, additional pathways are being exploited toward the assessment of the factors involved in the initiation and progression of CRC $(15,16)$.

Finally, prevalence of such alterations may vary during the disease progression phases and among different types of metastasis, with putative changes in the biological behavior of secondary CRC lesions, including response to targeted treatments $(13,17)$. At present, KRAS mutation analysis is performed in nearly all cases via tissue samples obtained from primary CRCs. Metastatic CRC lesions are usually considered as qualitatively similar, or even identical to the primary tumors. The aim of this study was to evaluate the spectrum and distribution of KRAS mutations in a large collection of CRC tissues, while also evaluating the concordance of primary and metastatic lesions among the available paired specimens from the same patients.

\section{Materials and methods}

Samples. A total of 729 patients with histologically confirmed advanced CRC and who regularly participated in the follow-up programs at the University Hospital and Local Health Unit (Sassari, Italy) were included in the study. To avoid any bias, CRC patients were consecutively collected between April 2009 and June 2013. Patients were included regardless of age at diagnosis and disease characteristics. No CRC cases included in this series were associated with clinically relevant colorectal polyposis. Details of the 729 patients are shown in Table I.

A Sardinian origin was ascertained in all cases through genealogical studies; for all patients, the place of birth of their parents and grandparents was assessed in order to assign their geographical origin within the island. Clinical and pathological features for the assessment of the disease stage at diagnosis, as well as the onset age and tumor anatomical location were confirmed by medical records and/or pathology reports.

Formalin-fixed, paraffin-embedded (FFPE) tissue samples of the CRC patients were obtained from the archives of the University Hospital and Local Health Unit. Tissue samples were estimated to contain $\geq 70 \%$ neoplastic cells by light microscopy. All patients were informed about the aims of this study and prior to collection of the tissue samples, written informed consent was obtained. The study was reviewed and approved by the ethical review board of the University of Sassari (Sassari, Italy).

Mutation analysis. All tumor tissues were collected and processed at the laboratory of the Institute of Biomolecular Chemistry of Sassari (Sassari, Italy); genomic DNA was isolated from tissue sections using a standard protocol and DNA quality was assessed for each specimen. In particular, paraffin was removed from the FFPE samples by treatment with Bio-Clear (Bio-Optica, Milan, Italy) and DNA was purified using the QIAamp DNA FFPE tissue kit (Qiagen, Valencia, CA, USA).

The coding sequence and splice junctions of exons 2 and 3 in the KRAS gene (where all pathogenetic mutations occur), were screened for mutations by direct automated sequencing. Briefly, polymerase chain reaction (PCR) was performed on 25-50 ng of isolated genomic DNA in a 9,700 Thermal cycler (Applied Biosystems, Foster City, CA, USA); all PCR-amplified products were directly sequenced using an automated fluorescence-based cycle sequencer (ABIPRISM 3100; Applied Biosystems), as described in our previous study (18).

The primer sequences used were as follows: Forward, TGT GTG ACA TGT TCT AAT ATA GTC ACAT and reverse, GGT CCT GCA CCA GTA ATA TGC for KRAS exon 2; and forward, GAC TGT GTT TCT CCC TTCT and reverse, TGG CAA ATA CAC AAA GAA AG for KRAS exon 3. Protocols for the PCR-based assays were designed and optimized at the laboratory of the Institute of Biomolecular Chemistry at the National Research Council (Sassari, Italy), and are available upon request. A total amount of 25-50 ng of isolated genomic DNA was used for PCR assay, with $0.5 \mu \mathrm{M}$ of forward and reverse primers for each KRAS codon (2 and 3), $1.5 \mu \mathrm{M} \mathrm{MgCl}_{2}$, $0.2 \mu \mathrm{M}$ dNTPs and 1 unit of recombinant Taq DNA polymerase (GE Healthcare, Piscataway, NJ, USA). The basic PCR conditions were as follows: 38 cycles of denaturation at $94^{\circ} \mathrm{C}$ for $30 \mathrm{sec}$, primer annealing at $55-64^{\circ} \mathrm{C}$ (depending on the primer) for $30 \mathrm{sec}$, and polymerase extension at $72^{\circ} \mathrm{C}$ for $1 \mathrm{~min}$. All PCR reactions were terminated with a 7-minute extension at $72^{\circ} \mathrm{C}$.

Statistical analysis. Statistical analysis was performed using the statistical package SPSS version 7.5 for Windows (SPSS, Inc., Chicago, IL, USA) by Pearson's $\chi^{2}$ test. The exact coefficient for sample proportion analysis was performed to determine all significant parameters. $\mathrm{P}<0.05$ was considered to indicate a statistically significant different.

\section{Results}

Distribution of KRAS mutations. In total, 219 (30\%) KRAS mutations were identified among the 729 analyzed cases. The distribution of the mutations in relation to KRAS codons was as follows: $158(72.1 \%)$ in codon $12,44(20.1 \%)$ in codon 13,15 $(6.9 \%)$ in codon 61 , and two $(0.9 \%)$ in other codons.

For patient classification, KRAS mutations were detected in 208 of $690(30.1 \%)$ primary tumors and $11(28.2 \%)$ of 39 meta- 
Table I. Characteristics of the 729 colorectal cancer patients at diagnosis.

\begin{tabular}{lr}
\hline Characteristic & $\mathrm{n}(\%)$ \\
\hline Gender & \\
Male & $443(61)$ \\
Female & $286(39)$ \\
Tumor site & \\
Right-transverse colon & $261(36)$ \\
Left colon & $294(40)$ \\
Rectum & $174(24)$ \\
Disease stage & \\
II & $259(36)$ \\
III & $248(34)$ \\
IV & $222(30)$ \\
Tumor grading & \\
Well-differentiated & $86(12)$ \\
Moderately differentiated & $583(80)$ \\
Poorly differentiated & $60(8)$ \\
Age, years & \\
$<50$ & $83(11)$ \\
$50-69$ & $438(60)$ \\
$\geq 70$ & $208(29)$ \\
\hline
\end{tabular}

static tissue samples. The site of metastasis was the liver in 17 $(43.6 \%)$ cases, the lung in seven $(17.9 \%)$ cases, the peritoneum or omentum in six (15.4\%) cases, the regional lymph nodes in four $(10.2 \%)$ cases, and the pancreas and vagina in one case $(2.6 \%)$ each. In the remaining three $(7.7 \%)$ cases neoplastic tissue was obtained from pelvic recurrences.

Mutation patterns in paired samples. Among the 31 (4.3\%) patients who had paired samples of primary CRC and synchronous or asynchronous metastases, 28 (90.3\%) showed consistent mutation patterns between the primary tumors and metastatic lesions. Of the paired samples, the metastatic samples predominantly constituted visceral (liver and lung) lesions, while the three secondary lesions were pelvic recurrences. In nine $(29.0 \%)$ of the paired cases, KRAS mutations were detected; as depicted in Table II. Among the three (9.7\%) paired cases with discrepancies in the KRAS mutation pattern between primary and secondary tumors, one presented an additive mutation (Q61L) in the metastatic tissue, one carried different mutation types $(\mathrm{Q} 61 \mathrm{H}$ in the primitive lesion and G13V in the metastatic one), and one exhibited a mutated (Q61L) primary tumor and wild-type metastasis (Table II). Among the remaining 708 unpaired patients, one case $(0.1 \%)$ only presented coexistence of two KRAS mutations (G12D and $\mathrm{Q} 61 \mathrm{~L})$ in the same tumor tissue.

\section{Discussion}

Targeted therapies have been recently introduced in medical oncology for the treatment of several malignant neoplasias. The most diffused currently in use are tyrosin kinase
Table II. Types of KRAS mutation among patients in whom molecular analysis was performed in the primary and metastatic lesions.

\begin{tabular}{lll}
\hline $\begin{array}{l}\text { Patient } \\
\text { no. }\end{array}$ & Primary tumor & \\
\hline 1 & G13V & Metastasis \\
2 & G13D & G13V + Q61L \\
3 & G13D & G13D \\
4 & Q61H & G13D \\
5 & Q61L & G13V \\
6 & G12V & Wild-type \\
7 & G12D & G12V \\
8 & G13D & G12D \\
9 & G12D & G13D \\
\hline
\end{tabular}

Table III. KRAS mutation consistency between primary and metastatic lesions as depicted in the current literature.

\begin{tabular}{|c|c|c|c|}
\hline First author (ref.) & Year & $\begin{array}{l}\text { Paired cases } \\
\text { examined, } n\end{array}$ & Consistency, $\%$ \\
\hline Knijn (30) & 2011 & 305 & 96 \\
\hline Cejas (31) & 2009 & 110 & 94 \\
\hline Santini (32) & 2008 & 99 & 96 \\
\hline Etienne-Grimaldi (33) & 2008 & 48 & 100 \\
\hline Park (34) & 2011 & 17 & 76.5 \\
\hline Watanabe (35) & 2011 & 43 & 88 \\
\hline Molinari (36) & 2009 & 37 & 92 \\
\hline Loupakis (37) & 2009 & 43 & 93 \\
\hline Mariani (38) & 2010 & 38 & 97 \\
\hline Baldus (39) & 2010 & 20 & 90 \\
\hline Italiano $(40)$ & 2010 & 59 & 95 \\
\hline
\end{tabular}

inhibitors for EGFR-mutated lung adenocarcinomas, trastuzumab for HER2-negative breast cancer and cetuximab or panituximab for KRAS-mutated CRC (19). The use of targeted agents imposed the molecular testing of genes confirmed to be involved in the pathogenesis of tumors.

Molecular testing for the assessment of targeted treatment is generally performed on tissue obtained from the primary lesion. This is particularly true for CRC, as endoscopic methods are widely used and offer the possibility to obtain consistent neoplastic tissue samples. Molecular analysis on the primary tumor in metastatic patients was considered effective in the past, as metastases were supposed to maintain the biological features of the primary lesions. This concept was based on previous evidence that primary and metastatic lesions share numerous morphological and immunohistochemical features, allowing pathologists to obtain a diagnosis, and that proliferation rates were generally similar in primitive and secondary neoplastic lesions (20). More recent acquisitions guide to a new pathophysiological vision of tumorigenesis, characterized 
as a dynamic process that continuously changes and evolves during the progression of the disease (21).

These acquisitions are principally based on several differences identified within the primary mass itself, and within the primary tumor and its subsequent distant metastases. Morphological and immunohistochemical variability in the context of a primary tumor is very common. Different areas of proliferation, cell cycle arrest, epithelial differentiation, cell adhesion and dissemination are often detected in a single tumor (22). These differences may influence the outcomes of molecular analyses, considering that such analyses are generally performed on a limited fraction of tumor tissues. Studies confronting molecular analyses on different samples of the same tumor tissues are required to establish the manner in which intratumoral heterogeneity impacts the results of molecular tests. In the present series, which included 729 patients with advanced CRC, only two cases with intratumoral variability of the mutation status (coexistence of mutations in KRAS exons 2 and 3; G12D + Q61L and G13V +Q61L) were identified, accounting for the $0.3 \%$ of all examined cases.

Discrepancies between the primary and secondary neoplastic lesions have been reported for several types of tumors. HER 2, estrogen receptor and progesterone receptor (PR) expression in patients with advanced stage breast cancer has been investigated by Vignot et al (23); marked discrepancies were found for PR expression, however, no definitive conclusions could be drawn, particularly with regard to the clinical impact of such findings. Shah et al (24) studied the metastatic mutational evolution in a single case of lobular breast cancer, evidencing 19 novel non-synchronous coding mutations of the 32 found in the metastasis, while Ding et al (25) identified two de novo mutations in the metastasis of a patient affected with a basal-like carcinoma of the breast. With regard to the non-small cell lung cancer, the discordance rates of EGFR mutations vary between 10 and 54\%; however, these figures must be interpreted with caution, given the heterogeneity of methods employed in various studies (23). Two studies have investigated the genomes of primary and metastatic pancreatic tumors, evidencing clonal expansions in the metastatic tumors and suggesting that mutations in cancer-driving genes may occur later in oncogenesis, and may alter the biological behavior of the lesions $(26,27)$.

Concerning CRC, several studies have investigated genetic heterogeneity between primary and secondary lesions. Goranova et al (28) demonstrated variations in APC, KRAS and TP53 expression, suggesting a certain heterogeneity, as was evidenced in this study; one patient presented a discordant $K R A S$ mutation, while another carried an additional mutation. However, it is important to comprehend whether, as well as the manner in which this heterogeneity impacts on clinical practice. Neumann et al (29) examined KRAS mutational status in 879 primary CRC specimens and 139 metastasis specimens, but did not identify any significant discrepancies. Knijin et al (30), Cejas et al (31) and Santini et al (32) in their series of 305, 110 and 99 cases of paired primary and metastatic lesions, respectively, demonstrated consistency rates of $\leq 96.4 \%$ (30-32). Similar results were also found in numerous smaller series (Table III), suggesting that molecular heterogeneity in primary and secondary CRC does not impact on the current clinical practice, oriented to the identification of candidates for targeted therapies (33-40). A meta-analysis including a total of 19 publications with $\sim 1,100$ paired primary and secondary CRC tissues has recently confirmed an extremely high concordance of the KRAS genotype in primary and metastatic tumors, while also showing the highest rates of discrepancies in lymph node metastases as compared with correspondent primary CRC (41). Overall, either type of tumor tissue, primary or secondary CRC (with a putative exception for metastatic lymph nodes), could be useful as a source to detect $K R A S$ mutations in selecting patients to be addressed to anti-EGFR therapy.

The proportion of the discordant cases in the majority of reports published ranges between 0 and $12 \%$; three $(9.7 \%)$ paired samples out of 31 analyzed patients demonstrated discrepancies in KRAS mutation patterns between primary and secondary tumors in this study. In one discrepant case, a wild-type primary tumor and mutated metastasis, suggested that mutations in such a gene may be acquired during disease dissemination. In the other two discrepant cases, wild-type metastasis was observed in one patient with mutated primary tumors and a different mutation pattern was observed in one patient between the melanoma lesions $(\mathrm{Q} 61 \mathrm{H}$ in the primary and G13V in secondary tumor). Altogether, it could be speculated that discrepant metastases may depend on the molecular heterogeneity of the cell types in a limited subset of primary CRCs.

Although the current practice in the detection of KRAS mutations can be considered accurate, some aspects must be addressed in the future. The most relevant is to establish clear protocols in mutational analysis methods in order to avoid technical imprecisions and to accurately identify mutations that may play a key role in the metastatic process in small tissue samples of primary tumors. Employment of novel methods and technologies for molecular analysis will ameliorate the current knowledge on the clinical significance and utility of genetic modifications and discrepancies arising in the course of neoplastic diseases $(23,42)$. This is likely to improve clinical practice allowing the identification of the best treatment strategy for all patients.

In conclusion, the results of the current study suggest that a high concordance exists between the results of KRAS mutation analysis performed in primitive and metastatic or recurrent neoplastic tissues in primitive and metastatic, or recurrent neoplastic tissues of patients with advanced CRC. A small proportion of non-concordant cases may occur and may be attributed to additional molecular events that arise as the metastatic process advances. The techniques currently in use for mutation analysis offer globally high-quality results, contributing substantially in the correct application of targeted therapies in clinical practice.

\section{References}

1. Jemal A, Center MM, DeSantis C and Ward EM: Global patterns of cancer incidence and mortality rates and trends. Cancer Epidemiol Biomarkers Prev 19: 1893-1907, 2010.

2. Palmieri G, Paliogiannis P, Scognamillo F, et al: Colorectal cancer epidemiology in an area with a spontaneous screening program. Acta Medica Mediterr 29: 231-234, 2013.

3. National Cancer Institute:CancerTrends Progress Report-2011/2012 Update. http://progressreport.cancer.gov/doc_detail.asp?pid=1\&did $=2011 \&$ chid $=105 \&$ coid=1026\&mid=. Accessed December 5, 2013 . 
4. Yabroff KR, Mariotto AB, Feuer E and Brown ML: Projections of the costs associated with colorectal cancer care in the United States, 2000-2020. Health Econ 17: 947-959, 2008.

5. Edwards BK, Ward E, Kohler BA, et al: Annual report to the nation on the status of cancer, 1975-2006, featuring colorectal cancer trends and impact of interventions (risk factors, screening, and treatment) to reduce future rates. Cancer 116: 544-573, 2010.

6. Jorissen RN, Walker F, Pouliot N, et al: Epidermal growth factor receptor: mechanisms of activation and signaling. Exp Cell Res 284: 31-53, 2003.

7. Yuan ZX, Wang XY, Qin QY, et al: The prognostic role of BRAF mutation in metastatic colorectal cancer receiving anti-EGFR monoclonal antibodies: a meta-analysis. PLoS One 8: e65995, 2013.

8. Malumbres M and Barbacid M: RAS Oncogenes: the first 30 years. Nat Rev Cancer 3: 459-485, 2003.

9. Heinemann V, Stintzing S, Kirchner T, Boeck S and Jung A: Clinical relevance of EGFR- and KRAS-status in colorectal cancer patients treated with monoclonal antibodies directed against the EGFR. Cancer Treat Rev 35: 262-271, 2009.

10. Bardelli A and Siena S: Molecular mechanisms of resistance to cetuximab and panitumumab in colorectal cancer. J Clin Oncol 28: 1254-1261, 2010

11. Cunningham D, Humblet Y, Siena S, et al: Cetuximab monotherapy and cetuximab plus irinotecan in irinotecan-refractory metastatic colorectal cancer. N Engl J Med 351: 337-345, 2004.

12. Markowitz SD and Bertagnolli MM: Molecular basis of colorectal cancer. N Engl J Med 361: 2404-2460, 2009.

13. Peltomäki P: Mutations and epimutations in the origin of cancer. Exp Cell Res 318: 299-310, 2012.

14. Boland CR and Goel A: Microsatellite instability in colorectal cancer. Gastroenterology 138: 2073-2087, 2010.

15. Jiang L, Huang M, Wang L, et al: Overexpression of MEKK2 is associated with colorectal carcinogenesis. Oncol Lett 6 : 1333-1337, 2013

16. Barzi A, Lenz AM, Labonte MJ and Lenz HJ: Molecular pathways: estrogen pathway in colorectal cancer. Clin Cancer Res 19: 5842-5848, 2013

17. Vermaat JS, Nijman IJ, Koudijs MJ, et al: Primary colorectal cancers and their subsequent hepatic metastases are genetically different: implications for selection of patients for targeted treatment. Clin Cancer Res 18: 688-699, 2012.

18. Palomba G, Colombino M, Contu A, et al: Prevalence of KRAS, BRAF, and PIK3CA somatic mutations in patients with colorectal carcinoma may vary in the same population: clues from Sardinia. J Transl Med 10: 178, 2012.

19. Ciavarella S, Milano A, Dammacco F and Silvestris F: Targeted therapies in cancer. BioDrugs 24: 77-88, 2010.

20. Winawer SJ: Natural history of colorectal cancer. Am J Med 106 3S-6S, discussion, 50S-51S, 1999.

21. Swanton C: Intratumor heterogeneity: evolution through space and time. Cancer Res 72: 4875-4882, 2012.

22. Nassar A, Radhakrishnan A, Cabrero IA, Cotsonis GA and Cohen C: Intratumoral heterogeneity of immunohistochemical marker expression in breast carcinoma: a tissue microarray-based study. Appl Immunohistochem Mol Morphol 18: 433-441, 2010.

23. Vignot S, Besse B, André F, Spano JP and Soria JC: Discrepancies between primary tumor and metastasis: a literature review on clinically established biomarkers. Crit Rev Oncol Hematol 84: 301-313, 2012

24. Shah SP, Morin RD, Khattra J, et al: Mutational evolution in a lobular breast tumour profiled at single nucleotide resolution. Nature 461: 809-813, 2009.

25. Ding L, Ellis MJ, Li S, et al: Genome remodelling in a basal-like breast cancer metastasis and xenograft. Nature 464: 999-1005, 2010 .
26. Campbell PJ, Yachida S, Mudie LJ, et al: The patterns and dynamics of genomic instability in metastatic pancreatic cancer. Nature 467: 1109-1113, 2010

27. Yachida S, Jones S, Bozic I, et al: Distant metastasis occurs late during the genetic evolution of pancreatic cancer. Nature 467: $1114-1117,2010$

28. Goranova TE, Ohue M, Shimoharu Y and Kato K: Dynamics of cancer cell subpopulations in primary and metastatic colorectal tumors. Clin Exp Metastasis 28: 427-435, 2011.

29. Neumann J, Zeindl-Eberhart E, Kirchner T and Jung A Frequency and type of KRAS mutations in routine diagnostic analysis of metastatic colorectal cancer. Pathol Res Pract 205: 858-862, 2009.

30. Knijn N, Mekenkamp LJ, Klomp M, et al: KRAS mutation analysis: a comparison between primary tumours and matched liver metastases in 305 colorectal cancer patients. Br J Cancer 104: 1020-1026, 2011

31. Cejas P, López-Gómez M, Aguayo C, et al: KRAS mutations in primary colorectal cancer tumors and related metastases: a potential role in prediction of lung metastasis. PLoS One 4: e8199, 2009.

32. Santini D, Loupakis F, Vincenzi B, et al: High concordance of KRAS status between primary colorectal tumors and related metastatic sites: implications for clinical practice. Oncologist 13: 1270-1275, 2008

33. Etienne-Grimaldi MC, Formento JL, Francoual M, et al: K-Ras mutations and treatment outcome in colorectal cancer patients receiving exclusive fluoropyrimidine therapy. Clin Cancer Res 14: 4830-4835, 2008

34. Park JH, Han SW, Oh DY, et al: Analysis of KRAS, BRAF, PTEN, IGF1R, EGFR intron $1 \mathrm{CA}$ status in both primary tumors and paired metastases in determining benefit from cetuximab therapy in colon cancer. Cancer Chemother Pharmacol 68: 1045-1055, 2011.

35. Watanabe T, Kobunai T, Yamamoto Y, et al: Heterogeneity of KRAS status may explain the subset of discordant KRAS status between primary and metastatic colorectal cancer. Dis Colon Rectum 54: 1170-1178, 2011.

36. Molinari F, Martin V, Saletti P, et al: Differing deregulation of EGFR and downstream proteins in primary colorectal cancer and related metastatic sites may be clinically relevant. Br J Cancer 100: 1087-1094, 2009.

37. Loupakis F, Pollina L, Stasi I, et al: PTEN expression and KRAS mutations on primary tumors and metastases in the prediction of benefit from cetuximab plus irinotecan for patients with metastatic colorectal cancer. J Clin Oncol 27: 2622-2629, 2009.

38. Mariani P, Lae M, Degeorges A, et al: Concordant analysis of KRAS status in primary colon carcinoma and matched metastasis. Anticancer Res 30: 4229-4235, 2010

39. Baldus SE, Schaefer KL, Engers R, et al: Prevalence and heterogeneity of KRAS, BRAF, and PIK3CA mutations in primary colorectal adenocarcinomas and their corresponding metastases. Clin Cancer Res 16: 790-799, 2010.

40. Italiano A, Hostein I, Soubeyran I, et al: KRAS and BRAF mutational status in primary colorectal tumors and related metastatic sites: biological and clinical implications. Ann Surg Oncol 17: 1429-1434, 2010

41. Han CB, Li F, Ma JT and Zou HW: Concordant KRAS mutations in primary and metastatic colorectal cancer tissue specimens: a meta-analysis and systematic review. Cancer Invest 30: 741-747, 2012.

42. Tolmachev V, Stone-Elander S and Orlova A: Radiolabelled receptor-tyrosine-kinase targeting drugs for patient stratification and monitoring of therapy response: prospects and pitfalls. Lancet Oncol 11: 992-1000, 2010. 